\title{
Interval Power Flow Analysis Based on Type-II Fuzzy Logic Forecasting
}

\author{
Ruihua $\mathrm{Bi}^{1,{ }^{*}}$, Li Tao ${ }^{1}$, Dongli $\mathrm{Xu}^{1}$ and Xiang Wang ${ }^{2}$ \\ ${ }^{1}$ School of Electrical Engineering, Nanjing Institute of Technology, 211167, Nanjing \\ ${ }^{2}$ State Grid Ningxia Electrical Power Company, 750001, Yinchuan \\ ${ }^{*}$ Corresponding author
}

\begin{abstract}
In the traditional power flow analysis, the output of generator is controllable and certain. While due to the intermittence and randomness of new energy resources, the output of new energy generator is uncertain, traditional power flow calculation cannot deal with this kind of uncertain power flow problem, thus type-II fuzzy logic forecasting and interval arithmetic are applied to solve this uncertain problem in the paper. Type-II fuzzy logic forecasting is used to get the output of generator in a mathematical sense that the uncertain variables can be express as an interval form, then interval arithmetic is applied to calculate power flow. As one of interval arithmetic, Krawczyk-Moore method is applied in power flow calculation in this paper, which convergence does not need to get inverse of interval matrix, and the initial interval obtained by type-II fuzzy logic can improve the problems of early convergence, slow convergence and no convergence which are drawbacks of interval arithmetic. The Algorithm is tested on IEEE 30-bus system to validate the applicability.
\end{abstract}

Keywords-interval power flow; type-II fuzzy logic forecasting; interval arithmetic; power analysis

\section{INTRODUCTION}

With the development of the technology of new energy generators such as wind energy and photovoltaic energy, distributed generators has become an efficient way to meet the needs of load growth, pollution reduction, energy resource consumption reduction and more reliable electricity supply. But limited by geography, weather and any other factors, these new energy resources are intermittent and random, traditional power flow calculation can hardly deal with these uncertainty problems. Until now, three kinds of methods are proposed to solve uncertainty problems: 1) stochastic method [1, 2], for stochastic information, the way with probabilities is applied. 2) Fuzzy mathematics method [3, 4], fuzzy mathematics is used to establish power analysis models and fuzzy membership function is used to deal with uncertainties. 3) Interval algorithm, due to the output of generator obtained by forecasting is an interval number, the solution is an interval number, interval algorithm is an applicable way to solve the uncertain problem. Compared with stochastic methods, interval power flow analysis needs fewer calculations. And compared with fuzzy methods, interval algorithm is not influenced by fuzzy membership function which is given by people and thus its results are not influenced by people.

The interval algorithm was first presented by Moore in 1966 [5], and later it was applied in power analysis. In 1992, Wang firstly came up with the application of interval algorithm in power analysis, and compared power flow results calculated by interval algorithm, Monte Carlo simulation method and other stochastic methods [6]. And later, more and more people focus on the application of interval algorithm. Bisection method was used by PEI to solve the problems of early convergence, slow convergence and no convergence [7]. Interval algorithm was applied by Chaturvedi to solve three phase unbalanced radial distribution networks [8]. Affine arithmetic was added to interval algorithm by DING Tao to shorten the gap between the upper and lower boundaries which aims to the inner problem of interval algorithm-a large overestimation of boundaries [9]. The fuzzy set approach and interval arithmetic were integrated by D. DAS and a noniterative method was achieved [10].

This paper combines type-II fuzzy logic forecasting with interval algorithm to get the solution of uncertain power flow problems. Type-II fuzzy logic forecasting is first used to get the initial interval of generator outputs, then interval algorithm is used to solve nonlinear equations and magnitude and phase angle of bus voltage are finally obtained. Compared with stochastic methods, it needs fewer calculations. And compared with fuzzy approach, it is not influenced by fuzzy membership function which is given by people and thus its results are not influenced by people. Furthermore, compared with other method based on interval algorithm, it does not need as many calculations as bisection method, but it can also solve the problems of early convergence, slow convergence and no convergence.

\section{TYPE-II FUZZY LOGIC FORECASTING}

Many people has discussed type-II fuzzy logic and its application in forecasting $[11,12,13]$.

\section{A. Type-II Fuzzy Logic Sets}

A type-II fuzzy set expresses the non-deterministic truth degree with imprecision and uncertainty for an element that belongs to a set. A type-II fuzzy set denoted by $\tilde{A}$ is characterized by a type-II membership function $\mu_{\tilde{A}}(x, u)$, where $x \in X, u \in J_{X}^{u} \subseteq[0,1]$ and $0 \leq \mu_{\tilde{A}}(x, u) \leq 1$ defined in (1)

$$
\begin{aligned}
& \tilde{A}=\left\{\left(x, \mu_{\tilde{A}}(x)\right) \mid x \in X\right\} \\
& \tilde{A}=\left\{\left(x, u, \mu_{\tilde{A}}(x)\right) \mid \forall x \in X, \forall u \in J_{x}^{u} \subseteq[0,1]\right\}
\end{aligned}
$$


If $\tilde{A}$ is continuous, it is denoted in (2).

$$
\tilde{A}=\left\{\int_{x \in X}\left[\int_{u \in J_{x}^{u} \subseteq[0,1]} f_{x}(u) / u\right] / x\right\}
$$

where $\iint$ denotes the union of $x$ and $u$.

If $\tilde{A}$ is discrete then it is denoted by (3).

$$
\tilde{A}=\left\{\sum_{x \in X} \mu_{\tilde{A}}(x) / x\right\}=\left\{\sum_{i=1}^{N}\left[\sum_{k=1}^{M_{i}} f_{x_{i}}\left(u_{i k}\right) / u_{i k}\right] / x_{i}\right\}
$$

where $\sum \sum$ denotes the union of $\mathrm{x}$ and $\mathrm{u}$.

If $f_{x}(u)=1, \forall u \in\left\lfloor\underline{J}_{x}^{u}, \bar{J}_{x}^{u}\right\rfloor \subseteq[0,1]$ the type-II membership function $\mu_{\tilde{A}}(x, u)$ is expressed by one type- 1 inferior membership function. $\underline{J}_{x}^{u} \equiv \underline{\mu}_{A}(x)$ and one type-1 superior $\bar{J}_{x}^{u} \equiv \bar{\mu}_{A}(x)$, then it is called an interval type-II fuzzy set denoted by (4) and (5).

$$
\begin{gathered}
\tilde{A}=\left\{\begin{array}{l}
(x, u, 1) \mid \forall x \in X, \\
\forall u \in\left[\underline{\mu}_{A}(x), \bar{\mu}_{A}(x)\right] \subseteq[0,1]
\end{array}\right\} \\
\tilde{A}=\left\{\int_{x \in X}\left[\underset{x \in\left[\underline{\mu}_{A}(x), \tilde{\mu}_{A}(x) \subseteq[0,1]\right.}{\int 1 / u}\right] / x\right\}
\end{gathered}
$$

If $\tilde{A}$ is a type-II fuzzy Singleton, the membership function is defined by (6).

$$
\mu_{\tilde{A}}(x)=\left\{\begin{array}{cc}
1 / 1 & \text { six }=x^{\prime} \\
1 / 0 & \text { six } \neq x^{\prime}
\end{array}\right\}
$$

\section{B. Interval Type-II Fuzzy Logic Inference}

A typical type-II Mamdani system is consisted of fuzzifier, inference system, rules, defuzzifier and type-reducer. And their relationship is shown in Fig 1.

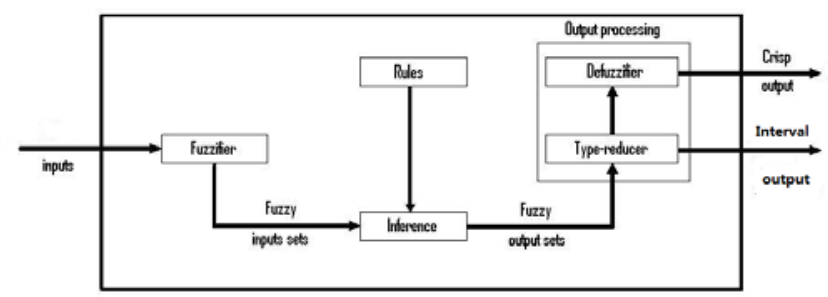

FIGURE I. TYPICAL TYPE-II MAMDANI SYSTEM

\section{INTERVAL POWER FLOW AlgORITHM}

Interval number has been used to describe uncertainties in many fields, and it has haven its own rules [14].

\section{A. Basic Rule}

An interval number $[\underline{x}, \bar{x}]$ is the set of real numbers $x$ such that $\underline{x} \leq x \leq \bar{x}$. Let $\mathrm{R}$ denote the set of real numbers. Then:

$$
x=[\underline{x}, \bar{x}]=\{\tilde{x} \in R \mid \underline{x} \leq \tilde{x} \leq \bar{x}\}
$$

where $\underline{x}$ is the lower boundary, and $\bar{x}$ is the upper boundary.

Mid point:

$$
m(X)=\min X=(\underline{x}+\bar{x}) / 2
$$

Width:

$$
W(X)=\bar{x}-\underline{x}
$$

And, if $X=[\underline{x}, \bar{x}], Y=[\underline{y}, \bar{y}] \in I(R)$, then we can define

$$
\begin{gathered}
X+Y=[\underline{x}+\underline{y}, \bar{x}+\bar{y}] \\
X-Y=[\underline{x}-\bar{y}, \bar{x}-\underline{y}] \\
X * Y=[\min (\underline{x} \underline{y}, \underline{y} \bar{x}, \bar{x} \bar{y}, \underline{x} \bar{y}), \max (\underline{x} \underline{y}, \underline{y} \bar{x}, \bar{x} \bar{y}, \underline{x} \bar{y})] \\
X / Y=[\underline{x}, \bar{x}] *[1 / \bar{y}, 1 / \underline{y}]
\end{gathered}
$$

\section{B. Krawczyk Operator}

Based on the researches of Moore [15], Krawczyk came up with Krawczyk-Moore iteration operator, which does not need to inverse interval matrix. The K-M operator is as below:

$$
K(y, \bar{x})=y-Y f(y)-\left(I-Y F^{\prime}(\bar{x})\right)(\bar{x}-y), \forall y \in \bar{x}
$$

Where, $Y$ is a nonsingular matrix with $n * n$ order; $I$ is identity matrix.

And Moore gave the way to choose $y$ and $Y$, and got the final iteration:

$$
\left\{\begin{array}{l}
\bar{x}^{k+1}=\bar{x}^{k} \cap K\left(\bar{x}^{k}\right) \\
K(y, \bar{x})=y-Y f(y)-\left(I-Y F^{\prime}(\bar{x})\right)(\bar{x}-y) \\
y^{k}=m\left(\bar{x}^{k}\right) \\
Y^{k}=\left(m\left(F^{\prime}\left(x^{k}\right)\right)^{-1}\right.
\end{array}\right.
$$

In fact, $y$ is a certain vector, and $Y$ is an inverse of certain vector. 


\section{Models of Power Flow Analysis}

Power flow analysis is the basic way to determine the state of power system based on topology of power system, parameters and boundary conditions, which is an essential part of power systems scheduling and running. It is the most basic and important calculation in power system analysis and base of system control and economic and safe analysis [16].

If there are $\mathrm{n}$ buses in a system, among which there are $\mathrm{m}$ PQ buses, then (n-m-1) PV buses, we can get equations below:

$$
\left[\begin{array}{l}
\Delta P \\
\Delta Q
\end{array}\right]=-\left[\begin{array}{ll}
H & N \\
K & L
\end{array}\right]\left[\begin{array}{l}
\Delta \theta \\
\Delta U
\end{array}\right]
$$

Where $\Delta \theta$ is correction of phase angle, and $\Delta U$ is correction of magnitude. $H, N, K, L$ is elements of Jacobian matrix.

$$
\begin{aligned}
& \Delta P_{i}=P_{i}-U_{i} \sum_{j=1}^{n} U_{j}\left(G_{i j} \cos \theta_{i j}+B_{i j} \sin \theta_{i j}\right) \\
& \Delta Q_{i}=Q_{i}-U_{i} \sum_{j=1}^{n} U_{j}\left(G_{i j} \sin \theta_{i j}-B_{i j} \cos \theta_{i j}\right)
\end{aligned}
$$

Where $G$ is real part of impedance, and $B$ is imaginary part of impedance. Index $i$ and $j$ denotes bus $i$ and bus $j$.

We apply interval numbers here, then:

$$
\begin{gathered}
\tilde{e}=\left[\begin{array}{c}
\tilde{\theta} \\
\tilde{U}
\end{array}\right]=\left[\left[\begin{array}{l}
\underline{\theta}, \bar{\theta}] \\
[\underline{U}, \bar{U}]] \\
F^{\prime}(\tilde{x})=-\left[\begin{array}{cc}
\tilde{H} & \tilde{N} \\
\tilde{K} & \tilde{L}
\end{array}\right]=-\left[[\underline{\underline{H}, \bar{H}}][\underline{\underline{K}}, \bar{K}]\left[\begin{array}{l}
\underline{N} \\
{[\underline{N}}
\end{array}\right]\right.
\end{array}\right]\right.
\end{gathered}
$$

$F^{\prime}(\tilde{x})$ is the interval extension of Jacobian matrix.

For example:

$$
\begin{gathered}
\tilde{H}_{i i}=\frac{\partial \Delta \tilde{P}_{i}}{\partial \tilde{\theta}_{i}}=\tilde{U}_{i} \sum_{j=1, j \neq i}^{n} \tilde{U}_{j}\left(G_{i j} \sin \tilde{\theta}_{i j}-B_{i j} \cos \tilde{\theta}_{i j}\right) \\
\tilde{H}_{i j}=\frac{\partial \Delta \tilde{P}_{i}}{\partial \tilde{\theta}_{j}}=-\tilde{U}_{i} \tilde{U}_{j}\left(G_{i j} \sin \tilde{\theta}_{i j}-B_{i j} \cos \tilde{\theta}_{i j}\right)
\end{gathered}
$$

When $Y^{(k)}=\left[m\left(F^{\prime}(\tilde{x})\right)\right]^{\prime}$, we need that $\left[m\left(F^{\prime}(\tilde{x})\right)\right]^{\prime}$ is not singular matrix, otherwise it would cause that the elements in $Y^{(k)}$ is not normal.

Then we can get the K-M operator:

$$
K=y^{k}-Y^{(k)} f\left(y^{k}\right)-\left[I-Y^{(k)} F^{\prime}(\tilde{x})\right]\left(\tilde{x}-y^{k}\right)
$$

Where $y^{k}=m(\tilde{x})$.

$$
f\left(y^{k}\right)=\left[\begin{array}{l}
P_{i s}-m\left(\tilde{U}_{i}\right) \sum_{j=1}^{n} m\left(\tilde{U}_{j}\right)\left(G_{i j} \cos \left(m\left(\tilde{\theta}_{i}\right)-m\left(\tilde{\theta}_{j}\right)\right)+B_{i j} \sin \left(m\left(\tilde{\theta}_{i}\right)-m\left(\tilde{\theta}_{j}\right)\right)\right. \\
Q_{i s}-m\left(\tilde{U}_{i}\right) \sum_{j=1}^{n} m\left(\tilde{U}_{j}\right)\left(G_{i j} \sin \left(m\left(\tilde{\theta}_{i}\right)-m\left(\tilde{\theta}_{j}\right)\right)-B_{i j} \cos \left(m\left(\tilde{\theta}_{i}\right)-m\left(\tilde{\theta}_{j}\right)\right)\right.
\end{array}\right]
$$

$$
Y^{(k)}=\left[m\left(F^{\prime}(\tilde{x})\right)\right]^{\prime}
$$

$I$ is an identity matrix, and using this operator to get solution:

$$
\left\{\begin{array}{l}
\tilde{x}^{(k+1)}=\tilde{x}^{(k)} \cap K^{(k)} \\
k=1,2, \cdots
\end{array}\right.
$$

Define terminal condition as $\left|\underline{x}^{k+1}-\underline{x}^{k}\right|<\omega$ and $\left|x^{k+1}-x^{-k}\right|<\omega$

\section{SOLVING PROCESS}

The flow chart of interval power flow analysis based on Type-II fuzzy logic forecasting is shown in Fig 2.

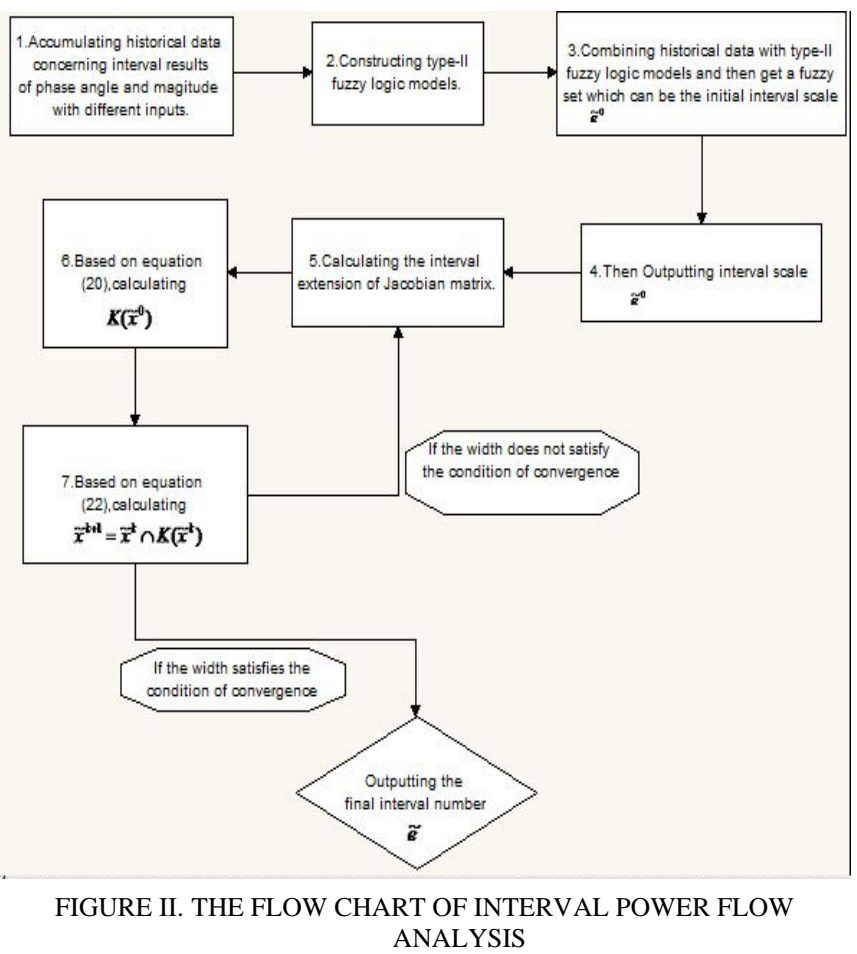

When type-II fuzzy logic models are constructed, the steps are as follows:

1) Designing fuzzy operator. Using Gauss Function with uncertain variance as its lower and upper membership degree 
function. And transferring one certain value to fuzzy sets. The function is as below:

$$
\begin{aligned}
& \bar{u}_{x_{k}}\left(x_{k}\right)=\exp \left[-\frac{1}{2}\left(\frac{x_{k}-x_{k}^{*}}{\bar{\sigma}_{k}}\right)^{\wedge} 2\right] \\
& \underline{u}_{x_{k}}\left(x_{k}\right)=\exp \left[-\frac{1}{2}\left(\frac{x_{k}-x_{k}^{*}}{\underline{\sigma}_{k}}\right) \wedge 2\right]
\end{aligned}
$$

where $x_{k}^{*}$ is the certain value, $\left[\underline{\sigma}_{k}, \bar{\sigma}_{k}\right]$ is the range of variance.

2) Constructing rules. Applying the IF-THEN and Mamdani . The rule is as below:

IF $x_{1}$ is $\tilde{F}_{1}^{l}$ and $\cdots \cdots x_{k}$ is $\tilde{F}_{k}^{l} \cdots \cdots$ and $x_{p}$ is $\tilde{F}_{p}^{l}$, then $y$ is $\tilde{G}^{l}$.

Where, $x_{k} \in X$ is the input of system, $\tilde{F}_{k}^{l}$ is the sets of ruling antecedents, $y \in Y$ is the ruling output, $\tilde{G}^{l}$ is the sets of consequent.

3) Constructing inference system. Using inputs and antecedents to produce activation sets. And using activation sets and consequent sets to calculate the output with its lower and upper membership degree function. The output form is as below:

$$
u_{\tilde{G}^{l}}(y)=\int_{b^{l} \in\left[\underline{f}^{l} * \underline{G}_{\tilde{G}^{l(y)}}, \bar{f}^{l} * \tilde{u}_{\tilde{G}^{l}(y)}\right.} \frac{1}{b^{l}} y \in Y
$$

where * is t-norm, $\bar{u} \tilde{G}^{I}(y) 、 \underline{u}_{\tilde{G}^{I}}(y)$ is the membership degree function of consequent. $\bar{f}^{l} 、 f^{l}$ is the membership degree function of activation sets.

4) Based on 2) and 3), we can get the inference model:

Rule1: if $x$ is $\widetilde{A}_{1}$ and $u$ is $\widetilde{B}_{1}$ and $v$ is $\widetilde{C}_{1}$, then $y$ is $\widetilde{G}_{1}$

Rule2: if $x$ is $\tilde{A}_{2}$ and $u$ is $\widetilde{B}_{2}$ and $v$ is $\widetilde{C}_{2}$, then $y$ is $\tilde{G}_{2}$

facts: $x$ is $\tilde{A}^{*}$ and $u$ is $\widetilde{B}^{*}$ and $v$ is $\widetilde{C}^{*}$

consequent: $y$ is $\widetilde{G}^{*}$

Where $x, u, v$ is input, $y$ is the anticipated interval range. $\tilde{A}_{k} \widetilde{B}_{k} \widetilde{C}_{k}$ is sets of input, $\widetilde{G}_{k}$ is sets of anticipated interval. They are all type-II fuzzy sets.

And $\tilde{G}_{k}=y^{l}=\left[y^{l}, \vec{y}^{-l}\right]$, here $y^{l}=\left[y^{l}, \vec{y}^{-l}\right]$ is the initial interval $\tilde{e}^{0}$

Inference process is as below:

1) Calculating fit measure. Comparing facts and antecedent to get fit measure between facts and membership degree function of antecedent of inputs.
2) Calculating activation. Using AND-OR operator to integrate fit measures yielded before.

3) Calculating useful membership degree function of consequent. Using activation to intersect corresponding membership degree function of anticipated interval.

4) Calculating output. Synthesizing all useful membership degree function of consequent and get the final interval.

5) Designing type-reducer. Using centered sets drop method, and replace anticipated interval in different sets with barycenter, and calculating weighted mean of barycenter, and get the final interval. We can express as below:

$$
Y_{\cos }=[\underline{y}, \bar{y}]=\left[\frac{\sum_{l=1}^{L} \bar{f}^{l-l} \bar{y}^{l}+\sum_{l=L+1}^{M} \underline{f}^{l} \underline{y}^{l}}{\sum_{l=1}^{L} \bar{f}^{l}+\sum_{l=L+1}^{M} \underline{f}^{l}}, \frac{\sum_{\substack{l=1 \\ f^{\prime}}}^{R} \underline{f}^{l} \bar{y}^{l}+\sum_{\substack{l=L+1 \\(25)}}^{M} \bar{f}^{l} \bar{y}^{l}}{\sum_{l=R+1}^{R}+\sum^{M} \bar{f}^{l}}\right]
$$

Where $y^{l}, \bar{y}^{l}$ is the lower and upper boundaries of barycenter sets, and $\underline{f}^{l} 、 \bar{f}^{l}$ is the lower and upper boundaries of activation sets, $L$ 、 $R$ is threshold value.

\section{NUMERICAL RESULTS AND ANALYSIS}

This paper uses IEEE 30-bus system to simulation, and considers the uncertainties varying within $\pm 5 \%$. Comparing the results of interval power flow with the results of power flow without uncertainty, this paper verifies the correction of this algorithm. IEEE 30-bus system connection diagrams is Fig III. And the simulation program is based on MATLAB and INTLAB [17].

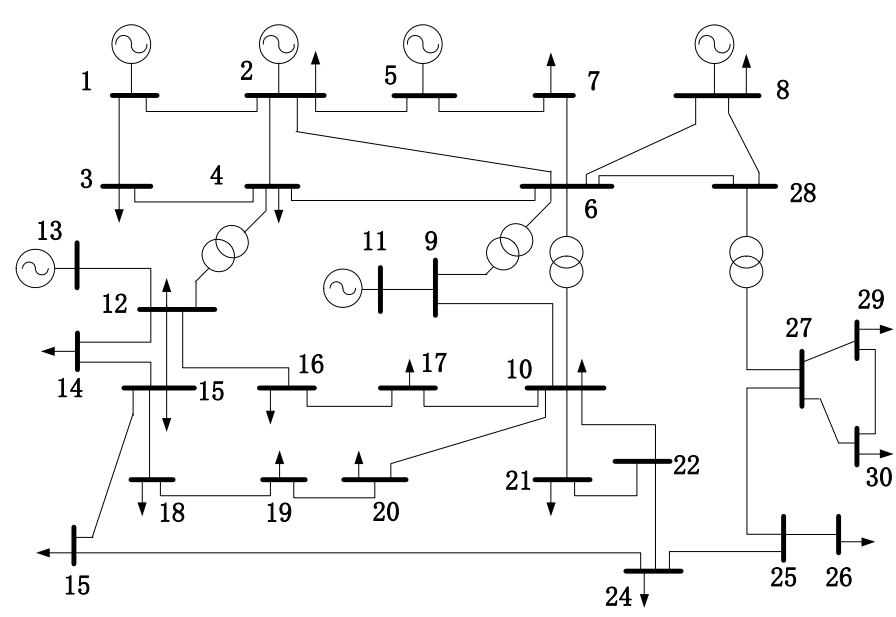

\section{Figure III. IEEE 30-BUS SYSTEM CONNECTION}

Table I gives the magnitude of voltage and phase angle of traditional power flow analysis and those based on type-II fuzzy forecasting and interval power flow analysis. 
TABLE I. STATE VARIABLES OF CERTAIN LOADS AND UNCERTAIN LOADS

\begin{tabular}{|c|c|c|c|c|}
\hline \multirow{2}{*}{ Num $^{a}$} & \multicolumn{2}{|c|}{ Traditional Power Flow } & \multicolumn{2}{|c|}{ Inter and type-II Fuzzy } \\
\hline & magnitude & Phase angle & {$[\underline{U}, \bar{U}]$} & {$[\underline{\theta}, \bar{\theta}]$} \\
\hline 1 & 0.9691 & -0.0201 & $\begin{array}{l}{[0.9670,0} \\
.9707]\end{array}$ & $\begin{array}{l}{[-0.0218,-} \\
0.0175]\end{array}$ \\
\hline 2 & 0.9706 & -0.0423 & $\begin{array}{l}{[0.9673,0} \\
.9711]\end{array}$ & $\begin{array}{l}{[-0.0439,-} \\
0.0393]\end{array}$ \\
\hline 3 & 0.9475 & -0.0384 & $\begin{array}{l}{[0.9455,0} \\
.9496]\end{array}$ & $\begin{array}{l}{[-0.0390,-} \\
0.0348]\end{array}$ \\
\hline 4 & 0.9746 & 0.0516 & $\begin{array}{l}{[0.9736,0} \\
.9774]\end{array}$ & {$[0.0495,0.0537]$} \\
\hline 5 & 0.9086 & -0.1147 & $\begin{array}{l}{[0.9065,0} \\
.9106]\end{array}$ & $\begin{array}{l}{[-0.1179,-} \\
0.1136]\end{array}$ \\
\hline 6 & 0.9353 & -0.0791 & $\begin{array}{l}{[0.9325,0} \\
.9365]\end{array}$ & $\begin{array}{l}{[-0.0806,-} \\
0.0762]\end{array}$ \\
\hline 7 & 1.0000 & 0.1563 & 1.0000 & {$[0.1526,0.1569]$} \\
\hline 8 & 1.0000 & 0.0313 & 1.0000 & {$[0.0274,0.0319]$} \\
\hline
\end{tabular}

And power of branches are shown in Table II.

TABLE II. POWER OF BRANCHES

\begin{tabular}{|c|c|}
\hline $\begin{array}{c}\text { Number of } \\
\text { branches }\end{array}$ & {$[\underline{S}, \bar{S}]$} \\
\hline $1-5$ & {$[-0.5871-0.3962 \mathrm{i},-0.4903-0.2994 \mathrm{i}]$} \\
\hline $1-6$ & {$[-0.3521-0.1931 \mathrm{i},-0.2669-0.1079 \mathrm{i}]$} \\
\hline $1-8$ & {$[0.6729+8.1023 \mathrm{i}, 0.9563+8.3856 \mathrm{i}]$} \\
\hline $4-6$ & {$[-0.7326-0.1771 \mathrm{i},-0.6401-0.0847 \mathrm{i}]$} \\
\hline $4-7$ & {$[1.4821+4.8957 \mathrm{i}, 1.7311+5.1448 \mathrm{i}]$} \\
\hline $2-9$ & {$[0.6443+15.2418 \mathrm{i}, 0.7911+15.3886 \mathrm{i}]$} \\
\hline $2-5$ & {$[-0.8030-0.4444 \mathrm{i},-0.6540-0.2953 \mathrm{i}]$} \\
\hline $2-3$ & {$[0.0697-0.3728 \mathrm{i}, 0.1135-0.1896 \mathrm{i}]$} \\
\hline $3-4$ & {$[0.8314+0.0105 \mathrm{i}, 1.0041+0.1622 \mathrm{i}]$} \\
\hline
\end{tabular}

In order to validate the correction of this algorithm, this paper takes advantage of certain flow calculations. In fact, we can divide the interval of outputs of generator into several discrete points. And the magnitude of voltage and phase angle can be calculated on the condition of these discrete points. Then based on the results of these certain flow, we can get the lower boundary and upper boundary of magnitude of voltage and phase angle. Then this range can be compared with the range obtained by algorithm which discussed by the paper, we can find range calculated by interval number includes the range calculated by certain numbers though the differences are very small. But the simulation using certain numbers, in fact, is far different from the calculation using interval algorithm. Obviously, calculation considering variable input and use of interval numbers are more close to real condition. This paper picks twenty certain power flows to calculate the interval of magnitude of voltage and phase angle. The results are shown in table 3 .

TABLE III. POWER FLOW RESULTS OF SIMULATION AND TYPE-II FUZZY FORECASTING

\begin{tabular}{|l|c|c|}
\hline \multirow{2}{*}{ Number of bus } & \multicolumn{2}{|c|}{ Interval and type-II Fuzzy Forecasting } \\
\cline { 2 - 3 } & {$[\underline{\underline{U}}, \bar{U}]$} & {$[\underline{\theta}, \bar{\theta}]$} \\
\hline 1 & {$[0.9670,0.9707]$} & {$[-0.0218,-0.0175]$} \\
\hline 2 & {$[0.9673,0.9711]$} & {$[-0.0439,-0.0393]$} \\
\hline 3 & {$[0.9455,0.9496]$} & {$[-0.0390,-0.0348]$} \\
\hline
\end{tabular}

\begin{tabular}{|c|c|c|}
\hline 4 & {$[0.9736,0.9774]$} & {$[0.0495,0.0537]$} \\
\hline 5 & {$[0.9065,0.9106]$} & {$[-0.1179,-0.1136]$} \\
\hline 6 & {$[0.9325,0.9365]$} & {$[-0.0806,-0.0762]$} \\
\hline 7 & 1.0000 & {$[0.1526,0.1569]$} \\
\hline 8 & 1.0000 & {$[0.0274,0.0319]$} \\
\hline Number of bus & \multicolumn{2}{|c|}{ Simulation of certain numbers } \\
\hline & {$[\underline{U}, \bar{U}]$} & {$[\underline{\theta}, \bar{\theta}]$} \\
\hline 1 & {$[0.9673,0.9703]$} & {$[-0.0203,-0.0184]$} \\
\hline 2 & {$[0.9677,0.9699]$} & {$[-0.0412,-0.0398]$} \\
\hline 3 & {$[0.9468,0.9479]$} & {$[-0.0372,-0.0361]$} \\
\hline 4 & {$[0.9741,0.9770]$} & {$[0.0501,0.0532]$} \\
\hline 5 & {$[0.9071,0.9098]$} & {$[-0.1168,-0.1152]$} \\
\hline 6 & {$[0.9331,0.9364]$} & {$[-0.0800,-0.0768]$} \\
\hline 7 & 1.0000 & {$[0.1533,0.1559]$} \\
\hline 8 & 1.0000 & {$[0.0280,0.0310]$} \\
\hline
\end{tabular}

The use of type-II fuzzy logic to find out the initial interval can solve the problem of convergence of interval algorithm. And due to this initial interval, the number of iterations is reduced. Because without this initial interval, the iterations would need lots of times of bisection to converge to this width.

\section{CONCLUSION}

Based on interval algorithm and type-II fuzzy logic, the paper discusses one way to get the solution of uncertain power flow problem. The application of interval number is close to real condition. The use of type-II fuzzy logic reduces iteration number and saves time which can meet the need of large system. The use of Krawczyk-Moore operator offers the iteration global convergence and it not only gives the solution but also considers estimation of error. Based on the results of simulation, this algorithm can successfully solve the problem of uncertainties, and do not have the problems of early convergence, slow convergence and no convergence.

\section{ACKNOWLEDGEMENTS}

This work was supported by State Grid Jiangsu Electric Power Company (Science \&Technology Innovation Project 281240426216058).

\section{REFERENCES}

[1] Borkowska B. Probabilistic load flow[J]. IEEE Transactions on Power Apparatus and Systems, 1974, 93(3): 752-759.

[2] VORSICJ MUZEK V, SKERBINEK G, Stochastic Load Flow Analysis [C]. Proceedings of the $6^{\text {th }}$ Mediterenean Electrotechnical Conference, May 22-24, 1991, Ljubana,Slovenia:1445-1448.

[3] Miranda V, Saraiva J T. Fuzzy Modeling of Power SUstem Optimal Load Flow[J]. IEEE Trans on Power Systems, 1992, 7(2):843 849.

[4] Das D, Ghosh S, Srinivas DK. Fuzzy Distribution Load Flow[J]. Electric Machines and Power Systems, 1999, 27(11):1215-1226.

[5] D. Pudjianto, C. Ramsay, G. Strbac. Virtual power plant and system integration of distributed energy resources[J]. Renewable Power Generation, IET, 2007, 1 (1): 10-16.

[6] Zian Wang, Fernando L. Alvarado. Interval Arithmetic in Power Flow Analysis[J]. Transactions on Power Systems, Vol. 7, No. 3, August 1992.

[7] Pei Ai-hua, LIU Ming-bo, ZHANG Chi, Interval algorithm for Power Flow Calculation with Uncertain Load[C]. Proceedings of the CSUEPSA, Vol. 16 No. 6, Dec. 2004. 
[8] A. Chaturvedi, K. Prasad, R. Ranjan, Use of Interval Arithmetic to Incorporate the Uncertainty of Load Demand For Radial Distribution System Analysis[J]. IEEE Transaction on Power Delivery, Vol. 21, No.2, April, 2006

[9] DING Tao, CUI Hantao, GU Wei, WAN Qiulan. An Uncertainty Power Flow Algorithm Based on Interval and Affine Arithmetic[J]. Automation of Electric Power Systems.

[10] D. DAS. A Noniterative Load Flow Algorithm for Radial Distribution Networks Using Fuzzy Set Approach and Interval Arithmetic[J]. Electric Power Components and Systems,33:59-72, 2005.

[11] Castillo O, Melin P. Comparison of hybrid intelligence systems, neural networks and interval type-II fuzzy logic for time series prediction [C]. IEEE International Joint Conference on Neural Networks, August 12-17, 2007, Orlando, USA, 2007: 3086-3091.

[12] Oscar Castillo, Patricia Melin. Computational Intelligence Software: type-II Fuzzy Logic and Modular Neural Networks[C]. 2208 International Joint Conference on Neural Networks (IJCNN 2008)

[13] ZHENG Gao, XIAO Jian. Forecasting study of power load based on interval type-II fuzzy logic method[J]. Electrical Machines and Control. Vol.16, No.9 Sep. 2012.

[14] Moore. R. E. Interval analysis[M], Englewood Cliffs, NJ, USA; Prentice Hall,1966

[15] Moore. R. E. Methods and applications of interval analysis[M], Philadelphia, SIAM,1979.

[16] Liu Jun, Liu Xuejun. The Application of MATLAB in Power System Analysis[] Proceedings of the EPSA, Vol.12, No.2, April 2000.

[17] http://www.ti3.tu-harburg.de/rump/intlab/ 